\title{
Les sources juridiques au service de l'histoire socio-culturelle de la France médiévale et moderne
}

\author{
Kouky Fianu/Sylvie Perrier
}

Depuis quelques décennies, l'histoire sociale, médiévale aussi bien que moderne, exploite ce matériau de choix que sont les archives judiciaires pour étudier le fait social dans toute sa complexité : travail, migrations, pratiques matrimoniales, marginalité, criminalité, etc. Depuis peu cependant, le questionnement s'est porté sur la nature et la signification des actes juridiques et sur les pratiques qu'ils révèlent. Le renouveau de l'histoire du notariat, en particulier, a amené les historiens à s'intéresser à la pratique notariale autant qu'au contenu des actes, redonnant ainsi sa juste place au contexte juridicoprofessionnel qui a produit à ces sources incontournables de l'histoire sociale'.

De son côté, l'histoire du droit s'est traditionnellement intéressée aux sources normatives (lois, coutumes) et a mis en évidence les évolutions des institutions juridiques (organes de législation, tribunaux, notariat, etc.) comme de la procédure. L'apport de l'anthropologie a récemment ouvert de nouveaux horizons en plaçant les utilisateurs et les agents de cette justice au centre du questionnement. L'anthropologie historique a également favorisé la comparaison des systèmes juridiques avec les sociétés non-occidentales et à travers le temps ${ }^{2}$. Une histoire des pratiques juridiques et de leur impact social est donc désormais possible.

Nos recherches respectives s'inspirent de ces approches pluridisciplinaires et cette parenté intellectuelle nous est vite apparue comme une invitation au dialogue. Au fil de nos conversations informelles, nous avons constaté que nous traitions des sources similaires, juridiques et para-juridiques, et que nous étions toutes deux sensibles à l'utilisation qui était faite de ces documents par les acteurs sociaux. Nous avons toutefois réalisé que nos démarches étaient distinctes, puisque nos questions de départ nous amenaient à explorer des univers tout à fait différents. Fortes de ce constat, nous avons décidé de tenter une expérience formelle et de nous engager dans un exercice comparatif. Quels résultats deux démarches différentes, mais basées sur des sources et une approche similaires peuvent-elles produire? Nous avons donc entrepris de décortiquer notre pratique historienne en comparant les diverses étapes de nos méthodes respectives ainsi que les résultats qui en sont issus.

Nous explorerons dans ce texte des pratiques sociales liées à la justice : d'une part, le rôle de l'écrit dans la société médiévale et, d'autre part,

\footnotetext{
1 Jean L. Laffont, "Introduction ", dans Notaires, notariat et société sous l'Ancien Régime, Toulouse, Presses universitaires du Mirail, 1990, p. 13 à 17.

${ }^{2}$ Voir en particulier Norbert Roulant, Anthropologie juridique, Paris, PUF, coll. « Droit fondamental ", 1988; Louis Assier-Andrieu, «L'anthropologie et la modernité du droit », Anthropologie et Société, vol. 13, n 1, 1989, p. 21 à 34.
} 
les recompositions familiales dans la société d'Ancien Régime. Ce faisant, nous chercherons à tracer des liens entre nos deux démarches pour souligner en quoi ce questionnement comparatif peut nous mener à une meilleure compréhension des rapports entre les individus et la justice aux époques qui nous intéressent. De plus, nous tenterons également de montrer en quoi cette expérience méthodologique a influencé nos deux recherches en cours.

\section{L'utilisation de la justice d'après des sources juridiques médiévales}

À partir du XII ${ }^{e}$ siècle, les établissements juridiques s'organisèrent et se multiplièrent dans le royaume de France: grands seigneurs, rois, villes, guildes, etc. se dotèrent d'institutions judiciaires, administratives et fiscales ${ }^{3}$. Cette évolution s'accompagna d'un écrit «conquérant ", l'administration du pouvoir (fiscalité, justice, etc.) reposant sur la production d'écrits authentiques, sur la constitution d'archives, et la justice exigeant de manière croissante des preuves écrites ${ }^{4}$. Dans la partie nord du royaume, où s'exerçait un droit coutumier, l'authentification des actes se traduisit le plus souvent par l'apposition du sceau d'une autorité, laïque ou cléricale, chargée d'attester la véracité et la légitimité des transactions inscrites dans les actes ${ }^{5}$. Dès la fin du $\mathrm{XII}^{e}$ siècle apparurent donc en France du Nord de nouvelles formes d'authentification des actes et de nouvelles pratiques de l'écrit, des pratiques qui se précisèrent avec le temps et que partagea à long terme l'ensemble de la population.

C'est dans ce contexte que s'insèrent les deux questions qui nous retiendront ici : comment se manifestèrent ces nouvelles pratiques? Comment et pour quelles raisons les individus faisaient-ils appel aux diverses instances responsables de la production d'un écrit authentique? L'étude qui suit repose essentiellement sur l'examen d'actes privés (ventes, donations, ententes, attestations, testaments, contrats d'apprentissage, etc.) émanant de juridictions gracieuses (tribunaux qui entérinent les transactions volontaires, non contentieuses) d'Orléans entre les $\mathrm{XIII}^{\mathrm{e}}$ et $\mathrm{XV}^{\mathrm{e}}$ siècles. $\mathrm{A}$ ce corpus de base $s$ 'ajoute un fonds d'inventaires après décès parisiens du $X V^{e}$ siècle qui mentionnent les actes détenus par des chanoines de Notre-Dame au moment de leur trépas.

${ }^{3}$ Olivier Guillot, Albert Rigaudière et Yves Sassier, Pouvoirs et institutions dans la France médiévale, tome II : Des temps féodaux aux temps de l'État, Paris, Armand Colin, 1994.

${ }^{4}$ Raoul C. van Caenegem, « La preuve dans le droit du moyen âge occidental - Rapport de synthèse ", dans La preuve, Bruxelles, Éditions de la librairie encyclopédique, 1965 (Recueils de la Société Jean Bodin pour l'histoire comparative des institutions, t. XVII), p. 691-753, plus particulièrement p. 742 et suivantes. Voir aussi Kouky Fianu et DeLloyd J. Guth (éd.), Écrit et pouvoir dans les chancelleries médiévales: espace français, espace anglais, Louvain-la-Neuve, FIDEM, 1997.

${ }^{5}$ Robert-Henri Bautier, "L'authentification des actes privés dans la France médiévale : notariat public et juridiction gracieuse ", dans Notariado público y documento privado. de los origines al siglo XIV. Actas del VII Congresso international de diplomatica Valencia 1986, Valencia 1989, vol. II, p. $701-772$ (Repris dans R.-H. Bautier, Chartes, sceaux et chancelleries. Études de diplomatique et de sigillographie médiévales, Paris, École des chartes, 1990, vol. I, p. 269-340). 


\section{Les nouvelles pratiques de l'écrit juridique}

Dès la fin du XII ${ }^{\mathrm{e}}$ siècle, la justice ecclésiastique était la plus étendue et la mieux organisée du royaume, surpassant les autres cours, seigneuriales ou princières. Son apogée eut lieu au $\mathrm{XIII}^{\mathrm{e}}$ siècle: les laïcs recouraient aux tribunaux épiscopaux (officialités) pour des actions entre laïcs, en raison de la faiblesse des pouvoirs temporels et de l'efficacité d'une procédure ecclésiastique plus rationnelle 6 . Parallèlement, chaque diocèse développa un service d'écritures authentiques, munies du sceau de l'évêque ou de son juge délégué, l'official. À partir des années 1250 , des contestations, en nombre croissant, s'élevèrent de la part des pouvoirs seigneuriaux et royaux du Nord contre la main-mise des officialités sur la juridiction gracieuse ${ }^{7}$ et les juges royaux n'acceptèrent plus ce type de lettres que comme des demies-preuves dans leurs tribunaux. La raison de ces attaques est liée aux ambitions de pouvoirs de mieux en mieux organisés : le droit leur servait en effet à affirmer autorité et légitimité, mais également à capter une importante source de revenus, celle de la taxe du sceau en particulier et de la justice en général. Tout au long du XIII ${ }^{e}$ siècle, le pouvoir royal s'affirmait. En 1280, le roi Philippe III le Hardi, ordonna l'instauration d'un tabellionnage (bureau d'écritures) dans toutes les prévôtés du royaume : ainsi naquit la lettre de baillie ou de prévôté, dont le succès fut immédiat car elle avait force exécutoire dans tout le royaume et dans toute cour laïque (contrairement à la lettre d'officialité) ${ }^{8}$. Ces bureaux d'écritures reposaient sur le travail de notaires royaux (aussi appelés tabellions) chargés d'entendre les parties et de mettre leur transaction en forme dans un acte, avant qu'il ne fût scellé par un garde du sceau. À Orléans, comme dans les rares régions du Nord qui ont été étudiées ${ }^{9}$, on assiste à un basculement du recours aux juridictions gracieuses entre 1280 et 1300 : les officialités furent délaissées au profit des prévôtés et de leurs tabellions, chargés d'authentifier les actes privés. Comment expliquer ce phénomène? Les informations sont très minces, mais la réponse réside sans doute dans la procédure juridique : plus les tribunaux royaux accroissaient leur compétence au détriment de l'officialité et privilégiaient la lettre de prévôté sur toute autre, plus les justiciables étaient amenés à s'en munir en cas de procès. Cette réponse logique doit toutefois être

\footnotetext{
${ }^{6}$ Paul Fournier, Les officialités au Moyen Age (1180-1328), Paris, Plon, 1880, p. 94-97. La question de la rationalité de la procédure inquisitoire adoptée par l'Église au XIII siècle est remise en question depuis plusieurs années, comme le résume Bruno Lemesle, «Premiers jalons et mise en place d'une procédure d'enquête dans la région angevine (XI ${ }^{\mathrm{e}}-\mathrm{XIII}{ }^{\mathrm{e}}$ siècle) ", dans $\mathrm{B}$. Lemesle (dir.), La preuve en justice de l'Antiquité à nos jours, Rennes, PUR, 2003, p. 69-93, plus particulièrement p. 70-71.

${ }^{7}$ P. Fournier, op. cit, p. 188 : l'auteur cite le cas de porteurs de lettres d'officialités qui, à Tours en 1294, furent attaqués et forcés à les manger...

${ }^{8}$ Louis Carolus-Barré, «L'ordonnance de Philippe le Hardi et l'organisation de la juridiction gracieuse ", Bibliothèque de l'École des Chartes, vol. 96 (1935), p. 5-48. R.H. Bautier, op. cit, p. 765-767.

${ }^{9}$ L. Carolus-Barré, op. cit et «L'ordonnance de Philippe le Hardi sur la juridiction gracieuse et son application en Champagne, dès $1280 \%$, Revue historique de droit français et étranger, vol. 39 (1961), p. 296-303. Robert-Henri Bautier, «L'exercice de la juridiction gracieuse en Champagne, du milieu du XIII siècle à la fin du XVe siècle ", Bibliothèque de l'École des Chartes, vol. 116 (1958), p. 29-106.
} 
nuancée : la prévention ne semble pas être la seule raison du recours à une juridiction gracieuse.

\section{Le recours aux justices volontaires}

Il convient en premier lieu de rappeler qu'au Moyen Âge rien ne forçait un individu à solliciter un acte authentique. En cour, la preuve écrite était de force égale à la preuve par témoins, ce qui se conçoit aisément dans la mesure où tout acte public était par sa nature même un témoignage attesté par un tabellion ou un notaire; jusqu'au $\mathrm{XVI}^{\mathrm{e}}$ siècle, elle pouvait d'ailleurs être renversée par le témoignage oral puisque, comme l'affirment les juristes médiévaux, "témoins passent lettres ${ }^{10}$ ». L'utilisation d'une juridiction gracieuse (ou volontaire) répondait donc à la volonté d'y recourir. Volonté des deux parties ou de la plus puissante seulement? En tout cas le geste révèle un choix qui témoigne de préoccupations précises.

Puisque jusqu'au $X V^{e}$ siècle les juridictions gracieuses royales du Nord ne conservaient généralement pas trace des transactions, chaque partie était responsable de garder ses lettres précieusement en cas d'un recours ultérieur en justice. La sensibilité à l'écrit dans un tel contexte semble extrême : par exemple, l'inventaire après décès d'un chanoine parisien de 1468 révèle qu'il avait plus de 500 lettres dans plusieurs coffres au moment de son décès, certaines d'entre-elles remontant des décennies en arrière ${ }^{11}$. Les justiciables accumulaient donc les preuves de leurs transactions chez eux. Ces sources, on le devine, n'ont pas traversé les siècles : les médiévistes, contrairement hélas à leurs collègues modernistes, n'ont que très peu de chance de trouver le contenu de ces «trésors " personnels ou familiaux. Les rares cas qui subsistent laissent cependant deviner le caractère préventif d'une telle accumulation. En obtenant un acte, les parties venues devant le juge d'un tribunal volontaire s'assuraient de pouvoir démontrer leurs droits même lorsque les témoins ne seraient plus là pour témoigner. L'écrit, vecteur de mémoire, pouvait ainsi dire le droit et la propriété si jamais s'élevaient des contestations futures.

Un autre facteur susceptible d'expliquer l'utilisation des justices gracieuses est la réaction, rapide, des justiciables face à la procédure et à ses changements. Par exemple, la contestation seigneuriale contre la compétence de l'officialité, qui se manifeste dès le milieu du XIII ${ }^{e}$ siècle, se traduit exactement à la même époque par l'apparition de lettres de prévôté à Orléans, quelque trente ans avant l'ordonnance créant les tabellionnages royaux. Dans le cartulaire inédit de la Commanderie Saint-Marc d'Orléans qui couvre la période 1148 à 1434 , la première lettre de prévôté date de 1259 : il s'agit d'un acte, en français, passé devant deux prévôts et scellé du sceau de la prévôté ${ }^{12}$. L'année suivante, une quittance entre particuliers est produite en latin sous le

\footnotetext{
10 Jean-Philippe Lévy, "Le problème de la preuve dans les droits savants du Moyen Âge ", dans La preuve, Bruxelles, Éditions de la librairie encyclopédique, 1965 (Recueils de la Société Jean Bodin pour l'histoire comparative des institutions, t. XVII), p. 137-167, plus particulièrement p. 153-156.

${ }^{11}$ Archives nationales (France) [ci-après AN], S 851B, $\mathrm{n}^{\circ} 21$, f. 16-44.

${ }^{12}$ AN, S $1010^{1}$, f. 22 , don de terres et de meubles de Jehanne la Paillarde à Saint-Marc (1259).
} 
sceau de la prévôté ${ }^{13}$ et, en 1264 , un acte de vente en latin est également produit par deux prévôts sous le sceau de la prévôté ${ }^{14}$. Les pratiques juridiques des particuliers s'ajustaient donc à la procédure en vigueur au moment de la transaction. C'est ainsi que l'on peut interpréter l'utilisation successive de plusieurs cours dans le cas de l'authentification d'une transaction entre les Templiers et le seigneur de Beaugency : garantie une première fois par le sceau du seigneur lui-même en 1233, la transaction fut authentifiée (sous forme de vidimus) en 1295 par les prévôts de Beaugency et scellée du sceau de la prévôté $^{15}$, puis authentifiée à nouveau en 1427 par la prévôté d'Orléans ${ }^{16}$.

Les particuliers étaient conscients du poids et de la valeur des sceaux : ils pouvaient recourir, pour une même transaction, à plus d'un bureau d'écriture, comme s'ils s'assuraient, en période de changements juridiques, d'avoir en main tous les instruments possibles de la preuve. En sollicitant une juridiction plutôt qu'une autre, les parties demandaient à être placées sous l'autorité d'un juge particulier: plus favorable, plus clément, plus efficace, incontournable, etc. L'élément économique ne doit pas être négligé non plus : les frais de sceau, variables d'une juridiction à une autre, pouvaient jouer un rôle déterminant dans le choix d'une institution plutôt que d'une autre. Les caractéristiques personnelles pouvaient également entrer en compte dans le choix effectué: certains individus avaient des actes pour la moindre transaction, tandis que d'autres se contentaient d'ententes verbales, comme le montre la comparaison des inventaires après décès d'Étienne de Montdidier et de Jean Oche, deux chanoines parisiens ${ }^{17}$. Enfin, les parties acceptaient de prendre plus ou moins de risque en optant pour une opération verbale ou un acte écrit.

Au-delà de ces aspects pragmatiques, de prévention, réaction et créativité, on voit également dans le recours à la juridiction gracieuse une dimension plus symbolique. On pouvait affirmer son statut juridique en imposant un tribunal à l'autre partie contractante (par exemple un clerc exigeant une lettre d'officialité pour une transaction avec un laïc). On pouvait également confirmer son statut social et signaler par l'usage que l'on faisait des tribunaux le type de relation que l'on entretenait avec la partie associée ${ }^{18}$, par exemple en obtenant une reconnaissance de dettes pour des montants infimes ou en n'en demandant pas pour des montants importants ${ }^{19}$. Enfin, affirmer la

\footnotetext{
${ }^{13} \mathrm{AN}, \mathrm{S} 1010^{1}, \mathrm{f} .67 \mathrm{v}$, quittance pour la vente entre particuliers d'une pièce (avril 1260).

${ }^{14}$ AN, S $1010^{1}$, f. 68 (août 1264).

${ }^{15} \mathrm{AN}, \mathrm{S} 1010^{1}$, f. $54 \mathrm{v}$ (décembre 1295).

${ }^{16} \mathrm{AN}, \mathrm{S} 1010^{1}$, f. 55 (novembre 1427).

${ }^{17}$ Étude effectuée dans Kouky Fianu, «Enregistrer la dette : le témoignage des sources de la justice gracieuse à Orléans $\left(\mathrm{XIII}^{\mathrm{e}}-\mathrm{XV}^{\mathrm{e}}\right.$ siècle) $)$, à paraitre dans Julie MayadeClaustre (dir.), Endettement privé et justice au Moyen Age, Paris, Publications de la Sorbonne.

${ }^{18}$ Craig Muldrew, The Economy of Obligation. The Culture of Credit and Social Relations in Early Modern England, Palgrave, 1998, chap. 8 et 9 en particulier, où l'auteur montre la place occupée par les tribunaux dans les relations sociales tournant autour du crédit et la réticence des justiciables à y recourir.

${ }^{19}$ K. Fianu, «Enregistrer la dette », op. cit.
} 
hiérarchie (la plus puissante des parties imposant son choix de juridiction ${ }^{20}$ ) ou l'appartenance (les universitaires forçant le recours au prévôt, gardien de leurs privilèges $^{21}$ ) pouvait se traduire par le recours à l'une ou à l'autre des juridictions gracieuses. En somme, l'utilisation de l'écrit authentique permettait d'exprimer des normes juridiques mais également des normes sociales. Aller chez le notaire revêtait aussi un aspect rituel, créateur de liens sociaux.

\section{La famille recomposée et la justice à l'époque moderne}

C'est dans le contexte du renforcement du pouvoir monarchique et de la mise en place de l'État moderne que s'est développée la structure documentaire désormais à la disposition de l'historien de la France prérévolutionnaire. La masse des sources juridiques dont disposent les modernistes n'est pas tant le produit d'innovations institutionnelles dans la France d'Ancien Régime que de l'effort du pouvoir royal pour uniformiser les procédures judiciaires et pour assurer un meilleur enregistrement des actes de la pratique ainsi que leur conservation. C'est tout d'abord par le biais des grandes ordonnances, dont les plus marquantes sont celle de Villers-Cotterêt (1539), le Code Louis (1667) et l'Ordonnance criminelle (1670), que les souverains et leurs principaux ministres ont mieux défini les compétences juridictionnelles et établi des procédures judiciaires valides pour l'ensemble du royaume ${ }^{22}$. Cette oeuvre législative fut complétée par les juristes qui ont expliqué toutes les finesses du système dans leurs nombreux traités de procédure ${ }^{23}$. Par ailleurs, la nécessité d'assurer la publicité des actes privés et les impératifs fiscaux ont amené le pouvoir royal à mettre en place des bureaux d'enregistrement des actes, insinuations laïques et contrôle des actes, et à produire des outils de recherche (tables de mariages, de tutelles, de testaments, de ventes, etc.) pour localiser rapidement les documents recherchés ${ }^{24}$.

${ }^{20}$ Bien qu'il n'ait pas étudié la question du rôle des justiciables dans le choix d'une juridiction ou d'une autre, R.-H. Bautier signale la forte compétition qui eut lieu en Champagne entre les pouvoirs ecclésiastiques et les autorités royales lorsque les «lettres de baillie" firent leur apparition. Voir R.-H. Bautier, "L'exercice de la juridiction gracieuse en Champagne », op. cit., p. 37.

${ }^{21}$ Serge Lusignan, "Vérité garde le roi »-La construction d'une identité universitaire en France (XIIF-XV siècle), Paris, Publications de la Sorbonne, 1999, p. 122-123.

${ }^{22}$ Jean-Marie Carbasse, Manuel d'introduction historique au droit, $2^{\mathrm{e}}$ éd., Paris, PUF, 2003 , p. 202-205; Bernard Barbiche, Les institutions de la monarchie française à l'époque moderne, $2^{\mathrm{e}}$ éd., Paris, PUF, 2001, p. 64-67.

${ }^{23}$ Claude de Ferrière, Le nouveau praticien contenant l'art de procéder dans les matières civiles, criminelles, et bénéficiales, suivant les nouvelles ordonnances, Paris, Denys Thierry et Jean Cochart, 1681; Jacques-Antoine Sallé, L'esprit des ordonnances de Louis $X I V$, Paris, Veuve Rouy/Knapen, 1755; Eustache Nicolas Pigeau, La procédure civile du Châtelet de Paris et de toutes les juridictions ordinaires du royaume, Paris, Veuve Desaint, 1779, 2 vol. Il existe également des «styles», sorte de guides de procédure à l'usage des praticiens.

${ }^{24}$ Marie-Françoise Limon, articles " Contrôle des actes » et " Insinuations laïques », dans Lucien Bély (dir.), Dictionnaire de l'Ancien Régime, Paris, PUF, 1996, p. 332-333 et 665-666; voir également Françoise Hildesheimer, "Insinuation, contrôle des actes et absolutisme ", Bibliothèque de l'école des Chartes, vol. 143, p. 163-164 et Gabrielle 
Cette mentalité procédurière se reflète dans les documents judiciaires produits par les institutions de la France d'Ancien Régime. L'exemple des comptes de tutelle des orphelins mineurs est particulièrement éloquent ${ }^{25}$. On y retrouve la transcription intégrale des actes relatifs à la tutelle (nomination du tuteur, avis de parents, décisions judiciaires) ainsi que des références précises à d'autres pièces utiles à la compréhension du compte (contrats de mariage, testaments, inventaires, etc.). Avec un peu de flair et beaucoup de patience, le chercheur peut retrouver ces documents parmi les milliers de mètres linéaires de sources juridiques conservées dans les divers dépôts d'archives français. Cela permet donc d'envisager une autre méthode que celle de ma collègue médiéviste, une approche qui place les acteurs sociaux au centre du projet.

Ce projet de recherche porte sur le remariage et les recompositions familiales dans la région de Toulouse au XVIII ${ }^{\mathfrak{e}}$ siècle. Pour mener à bien cette enquête, il a d'abord fallu constituer un échantillon de familles, tant urbaines que rurales, à partir d'un ensemble de contrats de mariage passés chez quatre notaires entre 1761 et $1770^{26}$. La seconde étape a consisté à rassembler les actes juridiques (actes notariés et procédures au civil) qui permettaient de baliser la trajectoire familiale pour mieux comprendre l'incidence du remariage dans l'expérience des familles de la France d'Ancien Régime. En se plaçant dans la perspective de ces familles toulousaines du XVIII ${ }^{\mathrm{e}}$ siècle, on peut reproduire un questionnement similaire à celui employẻ par ma collègue médiéviste : comment et pourquoi les familles utilisaient-elles la justice sous l'Ancien Régime?

De nombreux motifs poussaient alors les familles à avoir recours à des procédures juridiques. Ce choix n'était pas toujours libre, puisque les dispositions royales imposaient plusieurs démarches, notamment quand des mineurs, ou leur patrimoine, risquaient d'être mis en péril par une situation familiale instable. Ainsi, Anne Cornus, veuve de Jean Poux, dut-elle d'abord abandonner la tutelle de son fils Philippe, faire procéder à la nomination d'un nouveau tuteur et rendre son compte de tutelle en justice avant de pouvoir contracter une nouvelle union avec Jean-Antoine Ayrolle ${ }^{27}$. Les procédures requises étaient plus lourdes pour les femmes qui passaient en secondes noces

Vilar-Berrogain, Guide des recherches dans les fonds d'enregistrement sous l'Ancien Régime, Paris, Imprimerie nationale, 1958, introduction.

${ }^{25}$ Sylvie Perrier, Des enfances protégées. La tutelle des mineurs en France (XVIT -XVIIT siècles), Saint-Denis, Presses Universitaires de Vincennes, 1998, p. 41-46.

${ }^{26}$ Corpus global de 596 contrats de mariage passés entre 1761 et 1770 chez deux notaires de Toulouse (Archives départementales de la Haute-Garonne [ci-après ADHG], 3E26494 à 3E26498, minutes du notaire Jean-Pierre Richard; 3E7631 à 3E7640, notaire Jean Vidal) et chez deux notaires du village de St-Jory (ADHG, 3E20907 à 3E20909, notaire Jean Claverie Rapas; 3E20916 à 3E20919, notaire Jean-Blaise Marmond). Des sousensembles ont ensuite été constitués pour regrouper les contrats où l'un des conjoints avait été précédemment marié et les contrats où l'un des conjoints était issu d'une famille recomposée.

${ }^{27}$ ADHG, B Sénéchal/Audience (1761-1765) : sentence sur compte de tutelle présenté par Anne Cornus, $1^{\sigma \pi}$ juin $1764 ; 3 \mathrm{E} 7634$ : contrat de mariage de Jean-Antoine Ayrolle et Anne Comus, 19 juin 1764, notaire Jean Vidal. Après la mort de son premier mari, Anne Cornus avait aussi fait faire un inventaire puisque son fils héritier était alors mineur (3E 11939 : inventaire des biens de Jean Poux, 2 septembre 1763). 
que pour les veufs dans la même situation, la puissance paternelle n'étant pas troublée par le remariage du père, qui conservait la gestion du patrimoine familial quel que fût son état matrimonial.

Le contrat de mariage était la pierre angulaire du système matrimonial toulousain au XVIII ${ }^{\mathrm{e}}$ siècle et même les conjoints les plus pauvres $\mathrm{y}$ avaient recours ${ }^{28}$. Répondant au besoin d'établir et/ou de spécifier les intérêts économiques des deux conjoints dans le futur ménage, le contrat de mariage était un acte complexe qui pouvait comporter de nombreuses clauses (composition et paiement de la dot, donation, gains de survie, institution d'héritier, etc.). Il avait un caractère préventif, puisque l'accord des deux familles sur certaines dispositions et le respect général des contrats de mariage dans la société toulousaine constituaient une garantie pour l'avenir. Mais il s'agissait également d'un acte juridique où les parties pouvaient se montrer très créatives, soit en s'écartant de la coutume locale, soit en ajoutant des dispositions particulières. Le contrat qui a uni le jeune Jean Bouisson, un garçon charron toujours mineur, et Jeanne Gauté, veuve d'un charron, illustre très bien comment les parties pouvaient s'écarter du modèle familial de préférence de leur société et établir leur propre pacte familial ${ }^{29}$. Normalement, selon la coutume de Toulouse, la fiancée apportait une dot que recevait le futur époux, qui devait par la suite en garantir l'intégrité sur ses propres biens (hypothèque légale). Dans le cas de l'union Bouisson-Gauté, le contrat stipule que les époux habiteront chez la veuve et que la dot de celle-ci sera constituée par les meubles qui se trouvent dans la maison. D'autre part, la mère et le frère de l'époux ont fait une donation à ce dernier, aussitôt remise entre les mains de la veuve Gauté qui a hypothéqué ses biens en garantie de la somme. Le contrat respectait donc la coutume puisqu'il contenait une constitution dotale en bonne et due forme, mais la véritable dot était la donation des parents de l'époux : les rôles étaient ainsi inversés et c'est Jean Bouisson qui entrait en position d'épouse dans la maison!

Les recompositions familiales donnaient aussi lieu à des situations où les membres non-apparentés de la famille reconstituée désiraient renforcer leurs liens de belle-parenté par des dispositions juridiques stabilisatrices. Ainsi, Pierre Roques, second époux de Jeanne Lassage, auparavant veuve de Jean Fagès, a choisi une solution originale pour resserrer les liens avec ses beaux-fils François et Jean Fagès. En effet, ils sont passés tous ensemble chez le notaire de St-Jory, en zone rurale près de Toulouse, pour faire écrire un acte de société les unissant à même pot et à même feu, pour profits et pertes ${ }^{30}$. Actes assez courants chez les métayers qui s'unissaient pour exploiter de vastes terres, de telles sociétés étaient très rares au sein des familles. Les Roques-Fagès ont

\footnotetext{
${ }^{28}$ Germain Sicard, « Comportements juridiques et société : les contrats de mariage avant et après la Révolution (Toulouse et pays toulousain) ", dans G. Sicard (dir.), Notaires, mariages, fortunes dans le Midi toulousain, Toulouse, Université des Sciences sociales de Toulouse, 1997, p. 89-153.

${ }^{29}$ ADHG 3E 7637 : contrat de mariage entre Jean Bouisson et Jeanne Gauté, 7 février 1767 (notaire Jean Vidal).

${ }^{30}$ ADHG 3E 20918 : acte de société entre Pierre Roques et Marguerite Lassage d'une part, et François et Jean Fagès d'autre part, 10 septembre 1767 (notaire Jean Blaise Marmond).
} 
poussé la logique jusqu'au bout puisqu'ils ont effectivement signé des baux de métairie ensemble et que les dots des filles du second lit ont été versées par la société et non pas par le père seul $1^{31}$.

Dans toutes les familles, et plus particulièrement dans les familles recomposées, vouloir prévenir d'éventuels conflits ou en régler certains qui existaient déjà étaient des motifs puissants pour avoir recours aux services des notaires et de la justice civile ${ }^{32}$. Les actes d'accord/transaction sont nombreux dans les minutes des notaires. C'est la voie choisie par certaines veuves pour régler leurs créances avec la famille de leur conjoint défunt pour pouvoir se reconstituer une dot afin de passer en secondes noces. C'est aussi le moyen emprunté par des cohéritiers, souvent de lits différents, pour régler les questions patrimoniales après le décès du père commun. Toutefois, ces querelles ne trouvaient pas toujours leur solution dans la discrétion de l'étude du notaire et les parties devaient alors recourir aux tribunaux civils pour régler leurs différends. Souvent utilisées stratégiquement pour faire pression sur l'adversaire, les procédures civiles n'arrivaient pas toujours à l'étape de la sentence définitive.

D'autres procédures juridiques avaient un caractère moins contentieux et si elles avaient une raison d'être très concrète, souvent dictée par les lois, elles comportaient également une part symbolique qu'il ne faut pas négliger. Ainsi, les pères, ou à leur défaut les mères, qui ne pouvaient être présents à la signature du contrat de mariage de leur enfant devaient se faire représenter par un procureur qui attestait de leur consentement à l'union. Cette situation était plus fréquente pour les mariages urbains, ce qui témoigne du fait que l'éloignement ne mettait pas fin à la puissance paternelle : tant les familles que les pouvoirs publics tenaient à ce que le consentement parental fût respecté. Autre procédure liée à la puissance paternelle, l'émancipation (qui à Toulouse pouvait être officialisée devant notaire contrairement aux autres régions du sud où celle-ci devait être faite en justice) avait une dimension économique et pratique, puisqu'elle permettait au fils de gérer ses biens et de faire des transactions publiques sous son propre nom. Mais elle avait également une importante dimension rituelle, les notaires décrivant la cérémonie où le fils agenouillé plaçait ses mains entre celles de son père et lui assurait respect et obéissance, malgré l'indépendance que le père lui concédait par acte juridique ${ }^{33}$.

Les familles recomposées de la France pré-révolutionnaire offrent donc une perspective intéressante pour étudier les pratiques sociales de la

${ }^{31}$ ADHG 3E20918 : contrat de mariage entre Geraud Ferail et Marie Roques, 27 juin 1767 (Marmond); contrat de mariage entre Pierre Blanc et Jeanne Roques, 4 juin 1769.

${ }^{32}$ Isabelle Carrier a montré récemment que les familles pouvaient également se servir de la justice civile pour entretenir, voire provoquer, des querelles et non pas seulement dans la perspective d'une résolution de conflit. I. Carrier, Virtuosité procédurière. Pratiques judiciaires à Montpellier au Grand Siècle, thèse de doctorat, Université McGill, 2003.

${ }^{33}$ Ainsi, devant le notaire Jean Vidal, François Touigne, domestique, émancipe-t-il son fils Jacques : " Et pour marquer ladite emancipation ledit Touigne pere a dejoint les mains et donné sa benediction a sondit fils ", ADHG, 3E7631 : contrat de mariage entre Jacques Touigne et Marie Roche, 21 novembre 1761. Les formules d'émancipation contiennent parfois aussi des références au droit civil et au droit divin. 
justice. Ces familles devaient souvent recourir à tout le potentiel que leur offraient les institutions judiciaires pour aplanir les difficultés créées par l'enchevêtrement des patrimoines à transmettre, pour assurer le bon fonctionnement de la cellule familiale malgré les intérêt divergeants des individus qui la composaient et pour protéger les plus faibles d'entre eux.

\section{En guise de conclusion}

Les résultats de l'expérience que nous avons tentée n'ont, bien entendu, pas la prétention d'offrir des conclusions générales aux innombrables questions que l'on peut se poser sur la justice médiévale ou d'Ancien régime. Mais ils reflètent un exercice méthodologique fort enrichissant à maints égards. Le tableau page suivante résume les deux projets, leurs similarités et leurs différences.

Les deux projets ont pour point de départ deux démarches différentes : dans un cas il s'agit de pister des actes privés pour comprendre des pratiques sociales de l'écrit juridique; dans l'autre, d'identifier des familles pour saisir des pratiques familiales d'utilisation de la justice. Chaque recherche est donc menée de façon totalement indépendante et s'articule dans un contexte historiographique qui lui est propre. Cependant, une approche méthodologique commune est rapidement venue bâtir des ponts entre ces projets. À une même méthode historique de base (constitution d'un corpus, mise en contexte et critique documentaire) s'ajoute un intérêt commun pour la norme juridique (institutions, juristes, etc.). Un questionnement similaire, portant sur les acteurs et les pratiques juridiques, nous rassemble finalement autour de la question de l'utilisation de la justice, dans une perspective d'anthropologie du droit. 
Tableau 1 : Comparaisons des démarches de recherche

\begin{tabular}{|c|c|c|}
\hline & K. FIANU & S. PERRIER \\
\hline DÉMARCHES & à partir d'actes privés & à partir de familles \\
\hline \multirow[t]{4}{*}{ RÉSULTATS } & $\begin{array}{l}\Rightarrow \\
\text { - de l'official au } \\
\text { tabellion royal }\end{array}$ & $\begin{array}{l}\text { ion } \\
\text { de la famille en crise à } \\
\text { la famille « normale » } \\
\text { grer des codes sociaux, }\end{array}$ \\
\hline & $\begin{array}{l}\quad \Rightarrow \text { rés } \\
\text { - ententes } \\
\text { (ex. : épuration de } \\
\text { comptes, contrats } \\
\text { d'apprentissages ) }\end{array}$ & $\begin{array}{l}\text { de conflit } \\
\text { accords } \\
\text { (ex. : transactions } \\
\text { entre une veuve et sa } \\
\text { belle-famille) } \\
\text { iser à l'avance des } \\
\text { nflictuelles }\end{array}$ \\
\hline & $\begin{array}{c}\qquad \Rightarrow p r \\
\begin{array}{l}\text { garantie de privilège } \\
\text { (ex. : vidimus) }\end{array} \\
\rightarrow \text { la justice est utilisée } \\
\text { prérogatives légales }\end{array}$ & $\begin{array}{l}\text { protection des veuves } \\
\text { et orphelins (ex.: } \\
\text { tutelle) } \\
\text { peler et conserver des }\end{array}$ \\
\hline & $\begin{array}{l}\qquad \Rightarrow \text { rit } \\
\text { - } \quad \text { affirmer la hiérarchi } \\
\text { ou l'appartenance }\end{array}$ & $\begin{array}{l}\text { soutenir la puissance } \\
\text { paternelle } \\
\text { (ex. : émancipation) } \\
\text { pouvoir symbolique }\end{array}$ \\
\hline
\end{tabular}

La principale observation à laquelle nous sommes parvenues au terme de l'exercice confirme que l'utilisation de la justice répond, dans les deux cas étudiés, à des besoins sociaux. Ce qui peut sembler ici une évidence mérite cependant d'être rappelé, dans la mesure où l'historiographie s'est longtemps attaché à comprendre les institutions judiciaires plutôt que leur interaction avec la société dans laquelle elles s'insèrent. La volonté de placer l'acteur social au cœur de cette justice est un phénomène assez récent et tributaire de la démarche inter-disciplinaire : l'anthropologie juridique associée 
à la discipline historique a permis d'éclairer le rôle des individus et des communautés dans la mise en place et le fonctionnement des institutions judiciaires.

Notre expérience a également mis en valeur la continuité pluriséculaire des phénomènes étudiés, nous permettant ainsi de mieux évaluer nos résultats respectifs, de donner à nos observations des interprétations plus nuancées, d'aller au-delà du cadre temporel qui nous est propre. Elle a permis de déborder des pratiques historiographiques parfois trop étroites et surtout de faire éclater la frontière si insidieuse qui malheureusement persiste entre l'étude du Moyen Âge et celle des Temps modernes. Si l'interdisciplinarité favorise l'enrichissement des approches et du questionnement, l'intradisciplinarité est sans aucun doute pour l'historien le meilleur moyen de préciser la validité de ses hypothèses.

Nous sommes cependant conscientes des pièges que peut présenter une telle démarche : elle est par définition construite sur un terrain d'enquête commun et tend à faire valoir des similitudes, au détriment des spécificités temporelles. Par exemple, le recours à des catégories contemporaines (donc communes) pour expliquer le passé peut entraîner des anachronismes si l'on n'y prête pas attention. Un regard critique permanent est donc de rigueur, tout comme il l'est dans la démarche interdisciplinaire ou, en tout état de cause, dans la démarche historique elle-même.

Les avantages de l'approche intradisciplinaire l'emportent nettement sur ses inconvénients. Outre l'apport personnel incontestable, nous avons voulu ici faire partager aux étudiants et aux collègues une expérience qui, en dépit de sa rareté dans le milieu académique, peut offrir de précieuses avenues méthodologiques à la recherche future. 